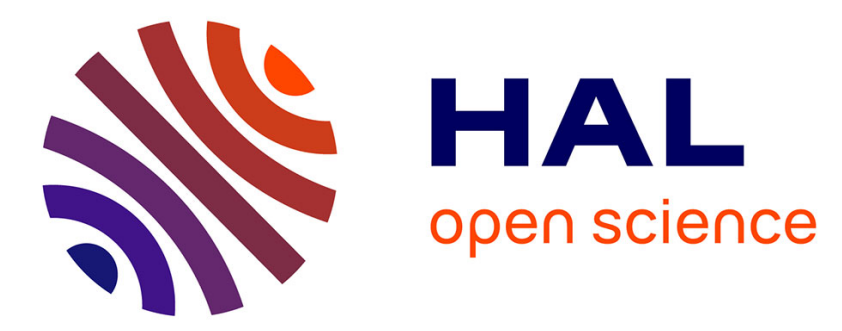

\title{
Self-organized homo-epitaxial growth of (001) vanadium assisted by oxygen surface reconstruction
}

\author{
Stéphane Andrieu, Pascal Turban, Bertrand Kierren
}

\section{To cite this version:}

Stéphane Andrieu, Pascal Turban, Bertrand Kierren. Self-organized homo-epitaxial growth of (001) vanadium assisted by oxygen surface reconstruction. Surface Science: A Journal Devoted to the Physics and Chemistry of Interfaces, 2016, 651, pp.154-163. 10.1016/j.susc.2016.04.008 . hal01372798

HAL Id: hal-01372798

\section{https://hal-univ-rennes1.archives-ouvertes.fr/hal-01372798}

Submitted on 17 Oct 2016

HAL is a multi-disciplinary open access archive for the deposit and dissemination of scientific research documents, whether they are published or not. The documents may come from teaching and research institutions in France or abroad, or from public or private research centers.
L'archive ouverte pluridisciplinaire HAL, est destinée au dépôt et à la diffusion de documents scientifiques de niveau recherche, publiés ou non, émanant des établissements d'enseignement et de recherche français ou étrangers, des laboratoires publics ou privés. 


\title{
Self-organized homo-epitaxial growth of (100) Vanadium assisted by Oxygen surface reconstruction
}

\author{
S. Andrieu ${ }^{1, *}$, P. Turban ${ }^{2}$, B. Kierren ${ }^{1}$ \\ 1 Institut Jean Lamour - Université de Lorraine / CNRS, 54506 Vandœuvre France \\ 2 Institut de Physique de Rennes - Université de Rennes I / CNRS, 35042 Rennes France
}

\begin{abstract}
In this paper the effect of oxygen on the vanadium homoepitaxial growth process is analyzed by using Auger spectroscopy, electron diffraction and scanning tunneling microscopy. As the oxygen induced 1x5 surface structure got a lattice spacing $6 \%$ different from the pure $\mathrm{V}$ lattice, relaxation is observed by electron diffraction during the growth. The average in-plane lattice spacing is thus shown to be proportional to the oxygen surface concentration. The surface lattice relaxation is observed to exponentially vary with the number of deposited atomic planes. A kinetic model is proposed and allows us to explain these observations. Furthermore, it helps us to distinguish two regimes depending on growth temperature. At high temperature, the oxygen surface concentration during growth is due to oxygen upward diffusion from the underneath $\mathrm{V}$ layer. For lower temperature however, this upward diffusion is not efficient and another source of oxygen contamination is evidenced. When the oxygen surface concentration is sufficient, a spectacular selforganization is observed at the surface by surface microscopy. Ribbons shape islands are observed and are tentatively explained as a consequence of oxygen surface concentration and stress induced by the surface reconstruction.
\end{abstract}

Keywords: molecular beam epitaxy; scanning tunneling microscopy; electron diffraction; vanadium; oxygen induced surface reconstruction; segregation; selforganized growth;

* corresponding author: stephane.andrieu@univ-lorraine.fr 


\section{1 -Introduction}

The understanding of the growth processes involved during thin films epitaxy is of particular interest in order to control the general properties of the synthesized system. The different growth modes, i.e. layer by layer (the Frank - Van der Merwe growth mode), three - dimensional (the 3D Volmer - Weber growth mode), and layer by layer followed by 3D islanding (the Stranski - Krastanov growth mode), have been established a long time ago and were currently observed on many various systems based on semi-conductors, oxides, metals etc...However, if these different growth processes may be justified by using the thermodynamics approach, the introduction of stress into the process is now well-known to be responsible for some peculiar behaviors according to theoretical prediction [1]. For instance, 3D growth was often observed on A/B systems for which a 2D growth was predicted by using thermodynamics consideration. This discrepancy may often be attributed to the occurrence of stress and strain. Self-organization on a surface is also a nice example of using stress to create self-assembled objects [1]. Different theoretical and experimental approaches were proposed to take into account the stress in predicting the growth mode or to use the stress in conceiving new functionalities and new devices (see [2] for a review). Among a large number of epitaxial systems, pure vanadium or vanadium based alloys are very interesting materials:

(i) V is not magnetic in bulk, but can become magnetic using alloying, stress or nanopatterning due to its $3 \mathrm{~d}$ valence electrons nature [3-5]

(ii) $\mathrm{V}$ is the pure element after $\mathrm{Nb}$ that has the highest superconducting temperature (5.4K) even for ultra-thin films [6]

(iii) vanadium oxides $\left(\mathrm{VO}, \mathrm{VO}_{2}, \mathrm{~V}_{2} \mathrm{O}_{3}, \mathrm{~V}_{2} \mathrm{O}_{5}\right)$ show peculiar electronic properties interesting for catalysis [7], or for insulator-metal transition that is not yet fully understood [8]

(iv) $\mathrm{V}$ intermixing with other metals takes place at high temperature $(850 \mathrm{~K}$ with Fe) giving some chance to use it as a robust buffer layer in spintronic devices for instance $[9,10]$. 
In this paper, we are interested in strain effect on the homo-epitaxial growth of Vanadium. It should be surprising that the strain could play a role during homoepitaxial growth. However, the vanadium surface is often "contaminated" by oxygen. Up to now, this contamination was attributed to diffusion from the bulk towards the surface during the surface smoothing. Vanadium surfaces free of oxygen are consequently very difficult to prepare [11-14]. This oxygen leads to very nice 1x5 $+5 \times 1$ surface reconstructions (and sometimes $1 \times n$ with $n \neq 5$ ) as observed by electron diffraction (LEED, RHEED) and Scanning Tunneling Microscopy (STM) [13, 15-17]. Moreover, we had clearly established by using RHEED that the Average In-Plane Lattice Spacing (noted AIPLS in the following) of this reconstructed surface is very different from the $\mathrm{V}$ bulk lattice spacing. Indeed, when growing pure $\mathrm{V}$ on top of such surfaces, some spectacular in-plane lattice relaxation was observed [12]. This large effect was attributed to the large mismatch between the lattice spacing of pure $\mathrm{V}$ and of the oxidized surface. This mismatch was estimated to be around $6 \%$. The $\mathrm{O}$ and $\mathrm{V}$ atomic arrangement and distances was analyzed by Koller et al [13] and Kralj et al [17] by using STM, and the expansion of the average lattice spacing from bulk V to $1 \times 5$ oxygen induced reconstruction was confirmed.

Consequently, speaking of homo-epitaxy of V on an oxygen induced (001) vanadium reconstructed surface is not pertinent. However, this mismatch obtained during the "homo"-epitaxial growth is a chance to analyze the growth by varying the temperature on a system with a large mismatch. Indeed, this analysis is often not possible in the case of a hetero-epitaxial metallic system since intermixing takes place when increasing the temperature. We thus examined the relaxation process of a growing $\mathrm{V}$ layer by electron diffraction varying the substrate temperature (noted $\mathrm{T}_{\text {sub }}$ in the figures) in the range $300-1020 \mathrm{~K}$. The corresponding surface morphology is observed by using STM after initiating the growth for different deposition temperatures. The growing islands shape was observed to be strongly anisotropic, which was tentatively explained by taking into account the strain induced by oxygen. The paper is divided in 4 parts. In the first part, the experimental details on the strain measurements, chemical analysis and STM analysis are reported. The strain experimental results are shown in the second part and the STM analysis in the third 
part. Finally, the original surface morphology observed by STM is discussed in the fourth part according to the strain observations.

\section{2 - Experimental details}

The vanadium buffer layers are grown by Molecular Beam Epitaxy (MBE) on (001) $\mathrm{MgO}$ substrates. The static pressure in the chamber was 7. 10-11 torr and decreased when cooling the cryogenic panels with liquid nitrogen. Vanadium was evaporated from a 99,5\% nominal purity target by using an electron gun, the pressure rising up to $4.10^{-9}$ torr during the process. The (001) bcc V epitaxy takes place on (001) $\mathrm{MgO}$ even at room temperature with the epitaxial relationship [110] (001) $\mathrm{MgO} / /$ [100] (001) V. In the following, the crystallographic directions are given in the BCC vanadium lattice. To get flat $\mathrm{V}$ surfaces, an annealing was performed up to $1000 \mathrm{~K}$. In these conditions, several surface superstructures may be observed, like the $1 \times 1$ (clean surface), and the pseudo $1 \times 1,5 \times 1$ and $6 \times 1$ [11, 13, 15, 17]. These surface arrangements were now clearly attributed to the presence of oxygen. Indeed, even if the oxygen contamination is very small during the growth process, this oxygen present in the bulk layer diffused towards the surface during the high temperature annealing (at least $1000 \mathrm{~K}$ ) necessary to smooth the surface. This is the reason why oxygen free (001) V surface are very difficult to obtain even after several ion bombardment cycles (see [16] and ref. therein). However, we managed to obtain clean buffer layers by growing the $V$ film at very low growth rates $(0.02 \mathrm{~nm} / \mathrm{s})$, limiting the thickness to 30nm (we have verified by X-Ray diffraction that the V layer is relaxed for such thickness), and using a moderate annealing temperature equal to $870 \mathrm{~K}$. To get oxygen on the surface, thicker buffer layers (100nm) are grown using higher growth rates $(0.2 \mathrm{~nm} / \mathrm{s})$ and annealed at high temperature $(970 \mathrm{~K})$ in order to get the $5 \times 1$ surface reconstruction induced by oxygen. The presence of oxygen was also checked by using Auger Electron Spectroscopy (AES). Note that with our Auger apparatus, we are able to separate the vanadium LMM peak at $509 \mathrm{eV}$ and the oxygen KLL peak at $512 \mathrm{eV}$ [15]. Such resolution was also reached by other groups [14] leading to similar results. 
The relaxation process was examined by using Reflection High Energy Electron Diffraction (RHEED) [12] operating with an acceleration voltage of $30 \mathrm{kV}$. During $\mathrm{V}$ deposition, a profile perpendicular to the streaks of the RHEED pattern in the [100] azimuth was recorded versus time. The $\mathrm{V}$ growth flux was thus fixed to 0.03 $\mathrm{nm} / \mathrm{s}$ during the recording. The diffraction experiments were performed at specular reflection and anti-Bragg geometry. We usually chose profiles with three streaks, which were fitted by lorentzian peaks. In order to improve the sensitivity on the lattice spacing measurement, we always measured the distance between (0-1) and (01) streaks on the RHEED pattern. The inverse of the distance between two peaks is proportional to the AIPLS. As an absolute determination of the in-plane lattice spacing is not easy due to technical limitations of the RHEED technique, we chose to set the initial distance between two streaks of the substrate surface to unity. Consequently, we only reported the relative variations of the AIPLS during the growth compared to this initial substrate in-plane lattice parameter. We thus not obtained an absolute measurement of the in-plane lattice spacing, since the in-plane lattice spacing of the initial surface could be different from the bulk, especially when a surface reconstruction takes place. With this technique, RHEED intensity, Full Width at Half Maximum (FWHM), and AIPLS variations versus deposition time were simultaneously obtained $[12,18-20]$. More details were reported in previous papers dedicated to this measurement procedure $[12,15]$.

STM measurements were performed at room temperature by using an Omicron STM1 apparatus operating in the constant current mode. W tips were used and prepared by $\mathrm{AC}$ electropolishing in $1 \mathrm{~N} \mathrm{NaOH}$ solution followed by heating in UHV using electron bombardment. The STM images were not corrected from the drift. Consequently, the real (001) V square lattice appeared to be distorted on the bare STM images. The [100] and [010] crystallographic directions can be determined easily in STM images for the following reasons: the single-crystalline $\mathrm{MgO}$ samples have a square shape and the sides are parallel to [100] and [010] MgO azimuths. The tip scanning in our microscope is inclined by $45^{\circ}$ from the sample holder sides so the sweeping is processed along $\mathrm{MgO}$ [110]. Finally, as the $\mathrm{V}$ on $\mathrm{MgO}$ epitaxial relationship is $\mathrm{V}[100](001) / / \mathrm{MgO}[110](001)$, the tip sweeping is along $\mathrm{V}[100]$. So 
according to the position of the $\mathrm{MgO}$ substrate on the sample holder, the crystallographic directions are indicated on the STM pictures in fig.4a to 9a.

\section{3 - Experimental results}

\section{1 - Surface relaxation measurements}

In a first series of experiments we looked at some possible link between the relaxation of the AIPLS during growth and the oxygen presence on the starting buffer surface (fig.1). For this purpose two different $V$ buffer layers were prepared, one without oxygen, the other with oxygen as checked by AES (fig.1a and d). This is confirmed by the RHEED patterns (fig1.b and e) showing the bcc (001) square lattice for the clean surface and $1 \times 5$ reconstruction for the oxidized surface (named as $1 \times 5-\mathrm{O}$ in the following). The AIPLS relaxation was thus examined during growth starting on these two surfaces (fig.1c and f). We clearly observed that the AIPLS of the deposited V layer tends to a different value $a_{f}$ than the initial AIPLS $a_{0}$ measured on the buffer layer. This relaxation process is however small starting from the clean surface, and became large for the oxidized one. Moreover, if the 5x1-O surface superstructure was observed on the initial buffer oxidized surface, it rapidly disappeared when growing the $\mathrm{V}$ film on top of it, leading to $1 \times 1$ RHEED patterns. The misfit between the initial buffer surface and V film was thus small starting with the buffer clean surface, and large with the 1x5-O reconstructed surface. This system is consequently similar to a hetero-epitaxial system with a misfit determined by the difference between the $5 \times 1$ and $1 \times 1$ surface lattice spacing. It should also be noted that this difference is not due to a change of the morphology (2D to 3D growth for instance) since RHEED oscillations were still observed during the growth process $[12,20]$.

At this stage it is worth asking what happened to oxygen during the $\mathrm{V}$ growth. For this purpose a series of 30ML thick $\mathrm{V}$ films were grown at different temperatures on a $1 \times 5$ surface reconstructed buffer layers. It should be noted that the starting 1x5 surface was always the same for each deposit: we just heated the sample up to $1020 \mathrm{~K}$ after one growth experiment and checked that we got the same oxygen quantity by AES and $1 \times 5$ by RHEED. For each experiment, AES was performed 
before and after the growth (fig. 2a and b). The oxygen surface (and subsurface since several monolayers under the surface are probed using AES) concentration was observed to decrease during the growth (inset in fig.2a and b), but this decrease went smaller when increasing the substrate temperature. At least, almost all the oxygen was moving to the surface for growth temperatures above $800 \mathrm{~K}$. This evidences that oxygen segregated to the surface during the growth process at least for high temperatures. The higher the growth temperature is, the greater the surface oxygen concentration. One consequence is that the after growth AIPLS $a_{f}$ (and therefore apparent misfit with $\mathrm{a}_{\mathrm{o}}$ ) was observed to decrease with the temperature of growth (fig.2c), since the surface at the end of the growth contains more oxygen when increasing the temperature. It should be noted that the $a_{f}$ error bars in fig.2c were due to possible fluctuation of the initial oxygen concentration on the $1 \times 5$ starting surface. Indeed, several authors reported the existence of the 1x5-O even if $\mathrm{O}$ surface concentration exceeds $1 \mathrm{ML}$ [17].

Another important feature is the particular shape of the AIPLS oscillations (fig.3). A logarithm plot of $\left\langle a(t)>-a_{f}\right.$ versus deposition time for one experiment showed that the relative variation of the AIPLS for each complete atomic plane exponentially decreased with time, as shown for different growth temperatures (inset in fig.3a and b). The oscillation envelop thus follows the law:

$$
\frac{<a(t)>}{a_{0}}-\frac{a_{f}}{a_{0}}=\left(1-\frac{a_{f}}{a_{0}}\right) \exp -\frac{t}{t_{0}}=\left(1-\frac{a_{f}}{a_{0}}\right) \exp -b n
$$

Where $t$ is the deposition time, $n$ is the number of deposited ML (with $n=v_{g r}$. $v_{g r}$ the growth rate in $\left.\mathrm{ML} / \mathrm{sec}\right), t_{0}$ is a characteristic time of the relaxation process and $b$ the associated term replacing the time deposition by the number of deposited atomic plane. This experimental law is justified in the following discussion part of the paper. Finally we should not work with time but rather with the thickness $n$ (in ML) since the growth rate $v_{g r}$ can slightly vary from one experiment to the other. The time to complete a monolayer (period of RHEED oscillations) was indeed observed to vary from 7 to $8 \mathrm{~s} / \mathrm{ML}$ from one experiment to the other. We thus measured the slope $b$ (in $\mathrm{ML}^{-1}$ ) for a series of experiment varying the growth 
temperature from 370 to $920 \mathrm{~K}$ (fig.3a, b, c). The variation of $\ln (b)$ with $1 / T_{\text {sub }}$ is reported in fig.3d. Two regimes are clearly observed: up to around 750K, this rate of relaxation is little dependent on the temperature, whereas it strongly increases above $750 \mathrm{~K}$. One should note that on some experiments done above $870 \mathrm{~K}$, the relaxation process is almost immediate and the relaxation slope is not measurable. These two regimes will be discussed in the following.

\section{2 - Surface imaging}

The STM analysis was first performed on the $5 \times 1$ reconstructed buffer layers (fig.4). Rectangular or square shape terraces were observed (fig.4a and b) reflecting the four-fold symmetry of the (001) square lattice. The average size of the terraces around 40nm with a large dispersion - was surprisingly not so high compared for instance to a similar (001) surface of bcc Fe prepared in the same system [21]. The O$5 \times 1$ organization consisted in bright (fig.4c) or dark lines (fig.4d) every 5 atomic distance -depending on the current and bias voltage applied on the tip $[15,17]$. The lines were interpreted as due to oxygen atoms on bridge sites, whereas oxygen atoms are in hollow sites between the lines as proposed by Koller et al [13]. Later, Kralj et al [17] observed by using STM other subtle arrangements suggesting 3-fold oxygen coordination consistent with our observations [15]. Finally, it is clear in all the studies that this surface reconstruction is due to oxygen. To look at any possible impact of oxygen on the growth process, a series of STM observation was thus performed after growing $0.5 \mathrm{ML}$ thick $\mathrm{V}$ submonolayers on $5 \times 1$ reconstructed $\mathrm{V}$ buffer layers for different substrate temperatures in the range $470-870 \mathrm{~K}$.

The beginning of $\mathrm{V}$ growth at $470 \mathrm{~K}$ is shown in figure 5 . The $\mathrm{O}-5 \times 1$ and $1 \times 5$ reconstructed terraces were still visible but bright spots were observed on these terraces. These bright spots are two-dimensional islands. The distribution of these islands is quite uniform on the surface and we have measured an average distance around $2 \mathrm{~nm}$ between them. The growth process thus originates from nucleation of 2D islands randomly distributed on the surface whatever the geometry of the initial terraces on the buffer layer surface (homogeneous nucleation, see ref. [22]). The shape of the growing islands were however not square, but rectangular and a little 
extended along [100] or [010] azimuths (Fig.5c and d). This revealed some inhomogeneous surface diffusion coefficients. The growth process was thus governed by homogeneous nucleation of elongated 2D islands in both [100] and [010] directions reflecting the four-fold symmetry.

The growing surface morphology significantly changed for deposition at 670, 770 and $870 \mathrm{~K}$ (fig.6, 7 and 8) compared to 470K. The terraces morphology of the initial buffer layer was no more visible and replaced by a regular distribution of ribbons elongated along [100] and [010] azimuths, again due to the four-fold symmetry of the square surface lattice. The distribution of the ribbons width was observed to decrease with the growth temperature: at $670 \mathrm{~K}$ (fig.6), the width of the ribbons varied from 5 to $11 \mathrm{~nm}$ and decreased down to 6 to $9 \mathrm{~nm}$ at 770K (fig.7), to finally spread between 6.5 to $7.5 \mathrm{~nm}$ at $870 \mathrm{~K}$ (fig.8). The distribution of the ribbons length varied in the same way. At $670 \mathrm{~K}$, the lengths of the ribbons were distributed from around $20 \mathrm{~nm}$ up to $80 \mathrm{~nm}$ (fig.6a). Such large distribution was still observed at $770 \mathrm{~K}$ but the average length was drastically increased (fig.7a). Indeed, some ribbons extended above 100nm long. This extension even increased at $870 \mathrm{~K}$, some ribbons being more than $150 \mathrm{~nm}$ long (fig.8a). If the appearance of growing ribbons was observed for the 3 temperatures, one may notice some differences regarding the nucleation process. At $670 \mathrm{~K}$, nucleation sites were observed on the ribbons, but also at the ribbons edges (fig.6c and d). This nucleation at ribbons edges was strongly reduced at $770 \mathrm{~K}$ (fig.7c and d) and almost disappeared at $870 \mathrm{~K}$ (fig.8c and d). The 2D islands nucleation on the ribbons was also efficient at $670 \mathrm{~K}$ and $770 \mathrm{~K}$, but is strongly attenuated at $870 \mathrm{~K}$. These behaviors were accompanied by recovering the oxygen induced surface reconstruction when increasing the growth temperature. If the oxygen rows, due to oxygen atoms on bridge sites, of the initial buffer layer cannot be observed for deposition at $670 \mathrm{~K}$ (fig.6c and d), some rows were observable at 770K (fig.7b), and became clearly visible at $870 \mathrm{~K}$ (fig.8b and c). It should be noted that these rows were systematically observed to be perpendicular to the ribbons (fig.7b, fig. $8 b)$.

Finally, this morphology was no more observed when growing the film at 1020K. The self-organized ribbons morphology disappeared. The surface 
morphology was observed to be close to the initial surface of the buffer layer. However, some similarities with the ribbons organization may be observed. If large terraces developed as shown in large scale images (fig.9a), some ribbons-like shape was still observed looking at lower scales (fig.9b and c), and the oxygen rows were still perpendicular to these ribbons. Another difference with the initial surface of the buffer layer has to be noticed: the oxygen rows characteristic of the O-1x5 surface reconstruction were no more well-organized on the terraces. The distance between the rows varied from 5 to 14 atomic distances (fig.9c and d). Finally, nucleation on the terraces was much less efficient compared to lower growth temperatures.

\section{4 - Discussion}

All these behaviors may be explained by noting that, according to our observations:

(i) Oxygen is present on the surface during the growth (fig.1 and 2)

(ii) The in-plane lattice spacing relaxation process is linked to the oxygen surface concentration (fig.1 and 2)

(iii) The average lattice spacing $a_{0}$ of the $0-1 \times 5$ surface structure is $6 \%$ larger than the pure $\mathrm{V}$ lattice spacing and thus induces stress on the growing V film.

Furthermore, the higher is the growth temperature, the higher the oxygen surface coverage and the lower the difference between the AIPLS $a_{f}$ at the end of the growth and the AIPLS $a_{0}$ of the starting $1 \times 5-\mathrm{O}$ reconstructed surface. Thus $a_{f}$ is completely determined by the oxygen concentration at this surface. As illustrated in fig.2c, we state that the AIPLS $\langle a(t)\rangle$ obtained after each atomic plane completion is proportional to the oxygen surface concentration $\theta(\mathrm{t})$. To find the corresponding linear equation we have two conditions: (i) at $\mathrm{t}=0$ we got the $\mathrm{O}-1 \times 5$ reconstruction of coverage $\theta_{0}$ with the AIPLS equal to $a_{0}$, and (ii) when $\theta(\mathrm{t})$ reaches zero, the surface is free of oxygen and the AIPLS is those of a pure V layer noted $a_{V}$ here. Thus:

$$
\langle\mathrm{a}(\mathrm{t})\rangle=\left(\mathrm{a}_{0}-\mathrm{a}_{\mathrm{V}}\right) \frac{\theta(\mathrm{t})}{\theta_{0}}+\mathrm{a}_{\mathrm{V}}
$$


In other words, the measurement of the AIPLS versus time after each atomic plane completion gives the oxygen surface coverage (in ML):

$$
\frac{\theta(\mathrm{t})}{\theta_{0}}=\frac{\langle\mathrm{a}(\mathrm{t})\rangle-\mathrm{a}_{\mathrm{V}}}{\mathrm{a}_{0}-\mathrm{a}_{\mathrm{V}}}
$$

So the measurement of the AIPLS versus time gives us some opportunity to determine $\theta(\mathrm{t})$. Moreover, with a set of experiments done by varying the growth temperature we may highlight the process(es) that supply this surface concentration during growth. We thus proposed the following analysis to account for the time dependence of the surface concentration. Considering the surface during growth, the following kinetic equation governs the oxygen concentration $\theta(\mathrm{t})$ at the growing surface (see for instance [23] and reference therein):

$$
\frac{\mathrm{d}}{\mathrm{dt}}\left(\frac{\theta(\mathrm{t})}{\theta_{0}}\right)=\mathrm{S} . \mathrm{F}_{\mathrm{O}}-\mathrm{F}_{\text {out }}=\left(1-\frac{\theta(\mathrm{t})}{\theta_{0}}\right) \mathrm{F}_{\mathrm{O}}-\mathrm{F}_{\text {inc }}-\mathrm{F}_{\mathrm{des}}
$$

where $F_{O}$ is the incoming fluxes of oxygen (in $\mathrm{ML} / \mathrm{s}$ ) on top of the surface during the growth and $F_{\text {out }}$ the flux of oxygen atoms leaving the growing surface. The oxygen flux living the surface $F_{\text {out }}$ contains desorption $F_{d e s}$ and incorporation of $\mathrm{O}$ during the growth $F_{\text {inc }}$ (incorporated oxygen actually leave the growing surface, see [23]). $S$ is the sticking coefficient (i.e. the probability to occupy free nucleation sites on the surface). The number of "free" sites on the surface is $\left(1-\theta / \theta_{0}\right)$. We assume that oxygen cannot go on a site already occupied so $S=\left(1-\theta / \theta_{0}\right)$. We can go one step further according to our problem by first eliminating desorption as evidenced by Shen el al [14] (consistent with the strong hybridization between $\mathrm{O}$ and $\mathrm{V}$ on the surface as calculated by two different groups [13,17]. Moreover, we consider that a part of the oxygen surface concentration $\alpha$ (incorporation probability) is incorporated during the time to complete a layer $\tau$ thus giving $F_{i n c}=\frac{\alpha}{\tau} \theta(t) / \theta_{0}$. Equation (4) becomes:

$$
\frac{\mathrm{d}}{\mathrm{dt}}\left(\frac{\theta(\mathrm{t})}{\theta_{0}}\right)=\left(1-\frac{\theta(\mathrm{t})}{\theta_{0}}\right) \mathrm{F}_{\mathrm{O}}-\frac{\alpha}{\tau} \frac{\theta(\mathrm{t})}{\theta_{0}}
$$

where $F_{O}$ is finally the total sources of oxygen that supply the oxygen surface concentration $\theta$. Even if this description seems very simplistic, this general equation 
accounts for all the processes happening at the surface during the growth. On the one hand the surface segregation happening at the surface by step climbing [23] is taken into account via the incorporation probability since if a $\alpha$ part is incorporated, it means that a $(1-\alpha)$ part stays on the surface (by climbing the steps or staying along the growing steps). On the other hand the bulk upward diffusion of oxygen towards the surface is included in $F_{O}$. The general solution of eq.(5) considering the initial conditions $\theta(t=0)=\theta_{0}$ is:

$$
\frac{\theta(\mathrm{t})}{\theta_{0}}=\frac{\mathrm{F}_{\mathrm{O}} \tau+\alpha \mathrm{e}^{-\frac{\mathrm{t}}{\tau^{\prime}}}}{\mathrm{F}_{\mathrm{O}} \tau+\alpha}=\frac{\langle\mathrm{a}(\mathrm{t})\rangle-\mathrm{a}_{\mathrm{V}}}{\mathrm{a}_{0}-\mathrm{a}_{\mathrm{V}}} \text { with } \frac{1}{\tau^{\prime}}=\mathrm{F}_{\mathrm{O}}+\frac{\alpha}{\tau}
$$

This justifies the exponential decrease with time observed on AIPLS during growth (Fig.3). This theoretical justification of eq.1 (deduced experimentally) also supports our statement on the proportionality between oxygen surface concentration and AIPLS. The characteristic decay time $\tau^{\prime}$ in the model can be compared to the experimental one deduced from figure 3 :

$$
\frac{1}{\tau \prime}=\frac{1}{t_{0}}=\frac{b}{\tau}=F_{0}+\frac{\alpha}{\tau}
$$

and the slope $b$ in eq.(1) is now shown to be a combination of incoming flux and incorporation flux. But we can go further by first calculating the surface coverage $\theta_{f}$ and the AIPLS $a_{f}$ at the end of a growth experiment $(t \rightarrow \infty)$ and second to link it to $\mathrm{F}_{\mathrm{O}}$ on the one hand and $\alpha$ on the other hand as:

$$
\frac{\theta_{\mathrm{f}}}{\theta_{0}}=\frac{\mathrm{F}_{\mathrm{O}} \tau}{\mathrm{F}_{\mathrm{O}} \tau+\alpha}=\frac{\mathrm{a}_{\mathrm{f}}-\mathrm{a}_{\mathrm{V}}}{\mathrm{a}_{0}-\mathrm{a}_{\mathrm{V}}}=\frac{\mathrm{F}_{\mathrm{O}} \tau}{\mathrm{b}}=1-\frac{\alpha}{\mathrm{b}}
$$

We can thus extract separately $F_{O}$ (the sources of incoming oxygen on the surface) and $\alpha$ (the incorporation probability) from both static (steady state leading to $a_{f}$ ) and dynamic (during the transient, slope $b$ ) experimental data as :

$$
\alpha=\frac{a_{0}-a_{f}}{a_{0}-a_{V}} \cdot b \quad \text { and } \quad F_{O} \tau=\frac{a_{f}-a_{V}}{a_{0}-a_{V}} \cdot b
$$

These two terms are plotted in Fig.10. The two regimes observed in fig.3c are now observed only on the $F_{O}$ variations and not on $\alpha$. The incorporation probability is thus around 5 to $20 \%$, varies slowly with temperature, and increases when 
decreasing the growth temperature as it should be. Two regimes are still observed on $\mathrm{F}_{\mathrm{O}} \tau$ variations, but now the signification is much clearer than from Fig.3. First, the activation energy of $F_{O}$ at high temperature $(>770 \mathrm{~K})$ is found to be equal to $1.25 \pm 0.1$ $\mathrm{eV} /$ atom $(120 \pm 10 \mathrm{KJ} / \mathrm{mol})$, very close to the activation energy of oxygen diffusion in bulk $\mathrm{V}$ reported by Shen et al. $\left(132 \mathrm{KJ} / \mathrm{mol}[14]\right.$. So this means that the $2^{\text {nd }}$ regime observed at high temperature corresponds to strong oxygen upward diffusion to the surface during growth. The situation is much different for the $1^{\text {st }}$ regime at lower temperatures $(\mathrm{T}<770 \mathrm{~K})$ : in that case the activation energy on $\mathrm{F}_{\mathrm{O}}$ drastically change and is close to $0.08 \mathrm{eV} /$ at. This means that another source of oxygen is present, and do not come from oxygen in the bulk underneath $\mathrm{V}$ layer. One possibility to explain this new source is to consider oxygen in the incoming flux coming from the evaporation of the $\mathrm{V}$ target. Indeed, during the evaporation process using an e-gun, the pressure in the chamber rise to around 5. 10-9 torr, and this flux contains mostly $\mathrm{V}$ atoms but also $\mathrm{O}$ atoms. Our estimation of $\mathrm{F}_{\mathrm{O}}$ allows us to estimate this flux compared to the $\mathrm{V}$ flux. From fig.10 we get an oxygen contamination flux $\mathrm{F}_{\mathrm{O}}=$ $0.006 \mathrm{ML} / \mathrm{s}$ at $370 \mathrm{~K}$ and $\mathrm{F}_{\mathrm{O}}=0.02 \mathrm{ML} / \mathrm{s}$ at $770 \mathrm{~K}$ to compare to the $\mathrm{V}$ flux chosen here around $0.2 \mathrm{ML} / \mathrm{s}$ (corresponding to $0.03 \mathrm{~nm} / \mathrm{s}$ ). This $\mathrm{O}$ contamination is larger than the purity degree of the $\mathrm{V}$ target $(99.5 \%)$ so we assume that it is also due to the pressure in the chamber during $\mathrm{V}$ evaporation. We thus consider that this oxygen supply on the surface during this first regime comes from the incoming evaporated total flux. The limited values of the incorporation probability in this "low" temperature regime mean that only a part of this oxygen is incorporated, the rest staying on the growing surface by surface segregation. Finally, the small activation energy of $F_{O}$ in this first regime suggests some dissociation mechanisms of molecules containing $\mathrm{O}\left(\mathrm{CO}\right.$ or $\left.\mathrm{CO}_{2}\right)$ that are strongly efficient at the $\mathrm{V}$ surface during growth. But the previous analysis does not allow us to go further.

In summary, our results show that the most reported assumption explaining oxygen segregation by bulk diffusion from the underneath $\mathrm{V}$ buffer layer up to the surface $[12-13,17]$ is correct for temperatures higher than $600 \mathrm{~K}$ without deposition [14], and contributes efficiently to rise oxygen surface concentration above $800 \mathrm{~K}$ during deposition at growth rates around $0.2 \mathrm{~nm} / \mathrm{s}$. One should conclude that below 
800K oxygen is buried by the vanadium growing layer. However, RHEED and AES results are not consistent with this assumption. Indeed even at moderate growth temperature we still observed a large quantity of oxygen by AES at the end of the growth (see fig.2a and b). Furthermore, if oxygen is totally buried, the in-plane lattice spacing at the end of the growth should lead to the $\mathrm{V}$ bulk value leading to a constant $a_{f}$ with temperature. This is not what we observed. Our analysis thus allows us to show that another source of oxygen contamination of the surface occurs in this regime. We suggest that some oxygen coming from the environment during $\mathrm{V}$ evaporation supply the $\mathrm{O}$ surface concentration. The surface oxygen supply in the first regime during the growth is thus a mixing of contamination from the incoming flux and surface segregation phenomenon.

This analysis shed some light on STM observations. Indeed, the surface morphology is driven by the ability to form the $1 \times 5$ and $5 \times 1$ domains on the surface depending on the temperature, oxygen surface concentration and kinetics. The growth process at $470 \mathrm{~K}$ is consistent with the limited $\mathrm{O}$ segregation during $\mathrm{V}$ growth that leads to homogeneous nucleation. One can note however that anisotropic surface diffusion is observed leading to elongated 2D islands for the very beginning growth process. We propose that this anisotropy is due to oxygen present on the initial buffer layer surface. Indeed, the oxygen surface reconstruction seems to be four-fold symmetric by RHEED or LEED but is in fact an assembly of $1 \times 5+5 \times 1$ reconstructed domains which are each 2-fold symmetric. The STM observations above $670 \mathrm{~K}$ are now consistent with increasing oxygen surface concentration since the nucleation is no more homogeneous and self-organization clearly takes place. The growth process gives rise to ribbons formation whose width distribution gets smaller and length longer when increasing the temperature. In parallel, the nucleation of $2 \mathrm{D}$ islands on top and at the edge of the ribbons still present at $670 \mathrm{~K}$ progressively disappears for higher growth temperatures. This self-organization is almost perfect at $870 \mathrm{~K}$ leading to very elongated ribbons up to $150 \mathrm{~nm}$ with a constant width around $7 \mathrm{~nm}$. This self-organization is consistent with oxygen accumulation at the surface since the oxygen atomic rows responsible for the $1 \times 5+5 \times 1$ superstructures (if no more observed below 700K) progressively reappear above 
$770 \mathrm{~K}$ growth temperatures. All these results show that oxygen is responsible for the self-organization via the tendency to form $1 \times 5-\mathrm{O}$ or $5 \times 1-\mathrm{O}$ domains. The question is how. One way is to consider the stress/strain induced by this surface reconstruction. As proposed by Koller et al [13] the surface oxygen arrangement is governed by the strong $\mathrm{V}-\mathrm{O}$ bonding that induces a strong tensile stress along the oxygen rows (perpendicular to the ribbons in our samples). On the opposite, the stress is partially release between oxygen rows (along the ribbons on our samples). We thus suggest that this tensile stress is at the origin of the ribbon shape by fixing the width to minimize the whole stress. Indeed, if the stress can be partly released in between the rows responsible for the $1 \times 5$, the stress is full along these rows. This may explain why the rows are perpendicular to the ribbons. Thus during growth vanadium atoms diffuse along the ribbons edge to nucleate at the end of the ribbons. The growth thus processes in ribbons with fixed width and extending along [100] and [010] azimuths. This self-organization during growth disappears at $1020 \mathrm{~K}$ due to massive oxygen diffusion at the surface leading to large $1 \times 5+5 \times 1$ (or $1 \times n$ ) reconstructed areas.

\section{5 - Conclusion}

During homoepitaxial growth of vanadium using molecular beam epitaxy, some lattice spacing relaxation is observed and clearly assigned here to oxygen on the growing surface. Using the RHEED measurements we show that the average surface lattice size is directly proportional to the oxygen surface concentration. Starting from this point, we model the kinetic evolution of oxygen surface concentration during the process. This analysis allows us to account for dynamic relaxation and to distinguish two sources of oxygen surface concentration supply that is backward diffusion at high temperature and incoming flux contamination at lower temperature. The peculiar self-organization observed during vanadium growth is shown to be linked to this oxygen present at the surface. The elongated shape of the two-dimensional islands is explains as a consequence of stress induced by the oxygen $1 \times 5+5 \times 1$ surface structure. Such well-organized islands on the surface may be used as model buffer layers for self-organization of preferential nucleation of adsorbed molecules for instance. 


\section{References}

[1] P. Muller, A. Saul, Elastic effect on surface physics, Surf. Sci. Rep. 54, 157 (2004)

[2] J. Narayan, Recent progress in thin film epitaxy across the misfit scale, Acta Materialia 61, 2703 (2013)

[3] H. Dreyssé, C. Demangeat, Theoretical approaches of magnetism of transition-metal thin films and nanostructures on semi-infinite substrate, Surf. Sci. Rep. 28, 65 (1997)

[4] Y. Huttel, G. van der Laan, T.K. Johal, N.D. Telling, P. Bencok, Induced V and reduced Co magnetic moments at the V/Co interface, Phys. Rev. B 68174405 (2003)

[5] T. Devolder, M. Manfrini, T. Hauet, S. Andrieu, Compositional dependence of the magnetic properties of epitaxial FeV/MgO thin films, Appl. Phys. Lett. 103, 242410 (2013)

[6] M. Gutsche, H. Kraus, J. Jochum, B. Kemmather, G. Gutekunst, Growth and characterization of epitaxial vanadium films, Thin Solid Films 248, 18 (1994)

[7] W. Gao, M. Li, R. Klie, E.I. Altman, Growth and characterization of model oxide catalysts, J. Elec. Spect. Rel. Phenom. 150, 136 (2007)

[8] see Y. Fagot-Revurat, M. Mehring and R. K. Kremer, Charge order driven spin-Peierls transition in a'-NaV2O5, Phys. Rev. Lett. 84, 4176 (2000) and reference therein.

[9] C.-H. Lambert, A. Rajanikanth, T. Hauet, S. Mangin, E.E. Fullerton, S. Andrieu, Quantifying perpendicular magnetic anisotropy at the Fe-MgO(001) interface Appl. Phys. Lett. 102, 122410 (2013)

[10] A.Rajanikanth, T.Hauet, F.Montaigne, S.Mangin, S.Andrieu, Magnetic anisotropy modified by electric field in V/Fe/MgO(001)/Fe epitaxial MTJs, Appl. Phys. Lett. 103, 062402 (2013)

[11] J.S. Foord, AP.C. Reed, R.M. Lambert, The (100) surfaces of chromium \& vanadium: Reconsiderations of their structure \& reactivity, Surf. Sci. 129, 79 (1983)

[12] P.Turban, L. Hennet, S. Andrieu, In-plane lattice spacing oscillations during $2 D$ hetero- and homo-epitaxy of metals Surf. Sci. 446, 241 (2000)

[13] R. Koller, W. Bergermayer, G. Bresse, F.L.D. Hebenstreit, C. Konvicka, M. Schmid, R. Podloucky, P. Varga, The structure of the oxygen induced (1x5) reconstruction of V(001) Surf. Sci. 480, 11 (2001)

[14] M. Shen, Q. Ma, I. Lee, F. Zaera, Oxygen Adsorption and Oxide Formation on V(100) Surfaces, J. Phys. Chem. C 111, 6033 (2007)

[15] F.Dulot, P.Turban, B.Kierren, J.Eugène, M.Alnot, S.Andrieu, (001)V surface structures analysed by RHEED and STM, Surf. Sci. 473, 172 (2001)

[16] B. Bischoff, C. Konvicka, A.J. Quinn, M. Schmid, J. Redinger, R. Podloucky, P. Varga, H. van Kempen, Scanning tunnelling spectroscopy on clean and contaminated V(001), Surf. Sci. 513, 9 (2002)

[17] M. Kralj, P. Pervan, M. Milun, K. Wandelt, D. Mandrino, M. Jenko, HRAES, STM and ARUPS study of (5x1) reconstructed V(001), Surf. Sci. 526, 166 (2003)

[18] J. Massies, N. Grandjean, Oscillations of the lattice-relaxation in layer-by-layer epitaxial growth of highly strained materials, Phys. Rev. Lett. 71, 1411 (1993) 
[19] J. Fassbender, U. May, B. Shirmer, R.M. Jungblut, B. Hillerbrands, G. Guntherodt, Oscillatory surface in-plane lattice spacing during growth of $\mathrm{Co}$ and of $\mathrm{Cu}$ on a $\mathrm{Cu}(111)$ single-crystal Phys. Rev. Lett. 751, 4476 (1995)

[20] P. Muller, P. Turban, L. Lapena, S. Andrieu, Elastic relaxation during 2D epitaxial growth: a study of in-plane lattice spacing oscillations Surf. Sci. 488, 52 (2001)

[21] V.Serin, S.Andrieu, R. Serra, F. Bonell, C.Tiusan, L. Calmels, M. Varela, J.S. Pennycook, E. Snoeck, M. Walls, C. Colliex, Atomic scale study of interfaces in epitaxial Fe/MgO/Fe magnetic tunnel junctions, Phys. Rev. B 79, 144413, (2009)

[22] see for instance "Crystal growth for beginners: fundamentals of nucleation, crystal growth and epitaxy" by I. Markov, World Scientific 1995

[23] S. Andrieu, F. Arnaud d'Avitaya, J.C. Pfister, Surface segregation mechanism during 2D epitaxial growth: the case of dopants in Si \& GaAs, J. Appl. Phys. 65, 2681 (1989) 


\section{Figure caption}

Figure 1: In-plane lattice spacing variation during growth at Tsub $=620 \mathrm{~K}$ depending on the initial oxygen surface coverage. On the left, almost no oxygen was detected by Auger spectroscopy (a) on the initial surface leading to a $1 \times 1$ RHEED pattern along the [100] direction (b) and small amplitude of the in-plane lattice spacing variations (c). On the right, the Auger initial oxygen coverage (d) leads to a 5x1 surface reconstruction as seen by RHEED along the [100] direction (e) and large amplitude of the in-plane lattice spacing variation (f).

Figure 2: Evolution of the oxygen quantity during V growth at $420 \mathrm{~K}$ (a) and $570 \mathrm{~K}$ (b) and link with the final relaxed in-plane lattice spacing (c). The oxygen quantity was measured before the growth and at the end of the growth by Auger spectroscopy (insert in a) and b), same scale as in fig.1). The final values of the in-plane lattice spacing observed after 30ML thick are plotted versus growth temperature in c).

Figure 3: In-plane lattice spacing evolution at 770K (a), 570K (b) and $370 \mathrm{~K}$ (c) and their logarithm dependence with time in insets. This logarithm variation is modelled via eq.(1) and allows to extract the attenuation coefficient $b$ (see text). $\ln (b)$ is plotted in (d) for a series of experiments at different growth temperatures where two regimes are evidenced.

Figure 4: (a) 200x200, (b) 100x100, (c) 40x40, (d) 8.5x8.5 nm2 raw STM images performed on the vanadium buffer layer before the growth (not corrected from the drift (a) V=-1.7 V, I=0.2 nA, (b) V=-0.45 V, I=1.67 nA, (c) V=-1.8 V, I=0.1 nA, (d) V=$0.45 \mathrm{~V}, \mathrm{I}=1.67 \mathrm{nA})$. The bright lines in (c) and dark lines in (d) are due to the $1 \times 5$ surface reconstruction.

Figure 5: (a) 200x200, (b) 100x100, (c) 50x50, (d) 30x30 nm2 raw STM images performed after $0.5 \mathrm{ML}$ thick $\mathrm{V}$ deposition at $470 \mathrm{~K}$ (not corrected from the drift, (a) to (d) V=-2 V, I=0.1 nA). The terraces of the initial buffer layer (like in fig.5) are still visible. The V 2D islands are much smaller than the size of the initial terraces and homogeneous nucleation is observed. 


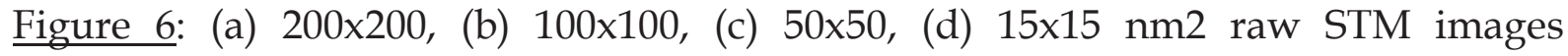
performed after $0.5 \mathrm{ML}$ thick $\mathrm{V}$ deposition at $670 \mathrm{~K}$ (not corrected from the drift, (a) and (b), V=-2 V, I=0.16 nA, (c) V=-1.4 V, I=0.15 nA, (d) V=-1.2 V, I=0.19 nA). The terraces of the initial buffer layer (like in fig.5) are no more visible. The growth is organized in long ribbons oriented along [01] or [10] directions depending on the reconstruction type, $1 \times 5$ or $5 \times 1$. Note some significant of the dispersion of the ribbons width at this temperature.

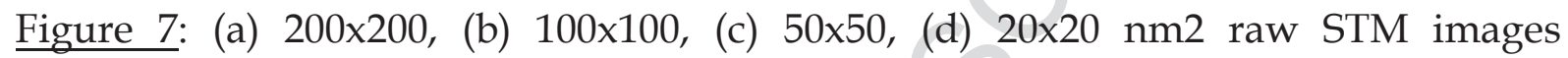
performed after $0.5 \mathrm{ML}$ thick $\mathrm{V}$ deposition at 770K (not corrected from the drift, (a) to (d) $\mathrm{V}=-1.9 \mathrm{~V}, \mathrm{I}=0.1 \mathrm{nA}$ ). The terraces of the initial buffer layer (like in fig.5) are no more visible. The dispersion of the lateral size of the growing ribbons is now very small.

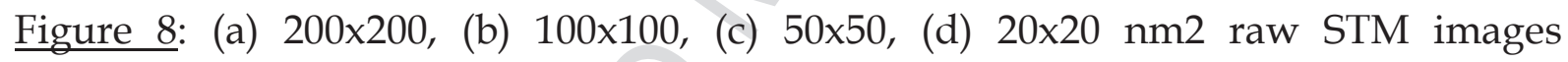
performed after $0.5 \mathrm{ML}$ thick $\mathrm{V}$ deposition at $870 \mathrm{~K}$ (not corrected from the drift, (a) $\mathrm{V}=-1.4 \mathrm{~V}, \mathrm{I}=0.17 \mathrm{nA},(\mathrm{b}) \mathrm{V}=-0.93 \mathrm{~V}, \mathrm{I}=0.45 \mathrm{nA}$, (c) and (d) V=.93 V, I=0.16 nA). The initial surface of the buffer layer is now totally reorganized in ribbons with a fixed lateral size. Note that the bright lines due to the $5 \times 1$ reconstruction visible in figure (b) and (c) -surrounded in black- are perpendicular to the ribbons.

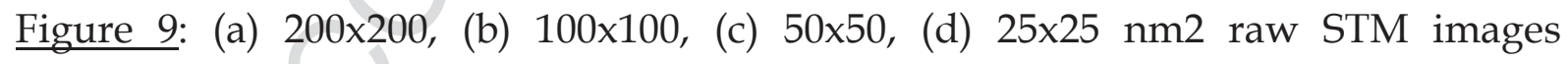
performed after $0.5 \mathrm{ML}$ thick $\mathrm{V}$ deposition at $1020 \mathrm{~K}$ (not corrected from the drift, (a) $\mathrm{V}=-1.1 \mathrm{~V}, \mathrm{I}=0.12 \mathrm{nA}$, (b) V=-1 V, I=0.2 nA, (c) V=-1 V, I=0.45 nA, (d) V=-1.5 V, I=0.1 $\mathrm{nA}$ ). The regular ribbons observed at lower temperatures have disappeared, and large reconstructed terraces develop to tend to the initial buffer layer morphology in fig.4.

Figure 10: Ln-plot of the oxygen supply $F_{O} \tau$ and incorporation probability $\alpha$ versus the inverse of growth temperature. These data are extracted -see text- from static data $a_{f}$ (fig.2c) and dynamic data $b$ (fig.3d). Two regimes are observed on the oxygen supply given by $F_{O} \tau$ : below $800 \mathrm{~K}$ the oxygen is supplied by incoming flux from the vanadium e-gun, and above $800 \mathrm{~K}$ oxygen upward diffusion from the $\mathrm{V}$ underlayer to the surface becomes efficient. 


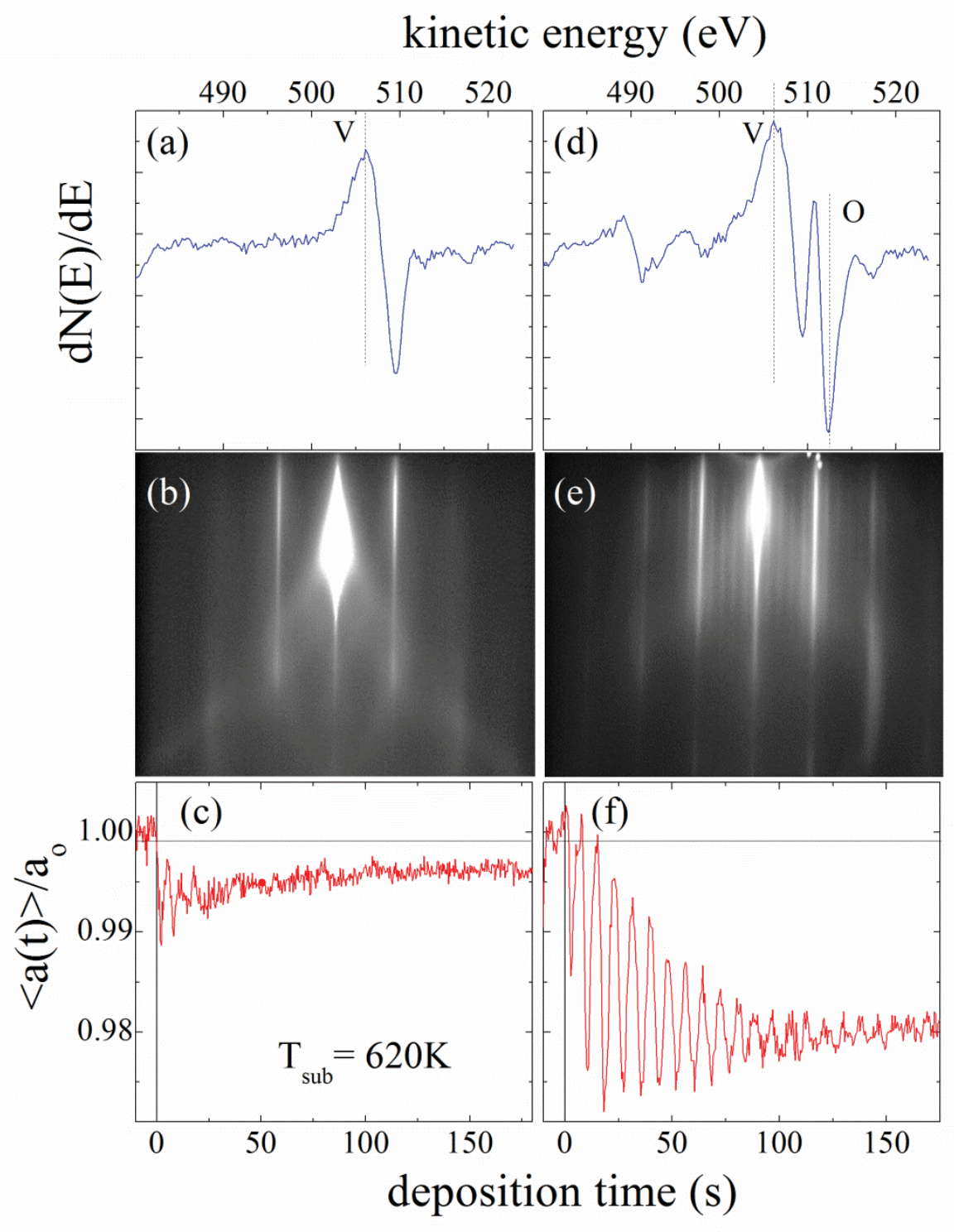

Figure 1 

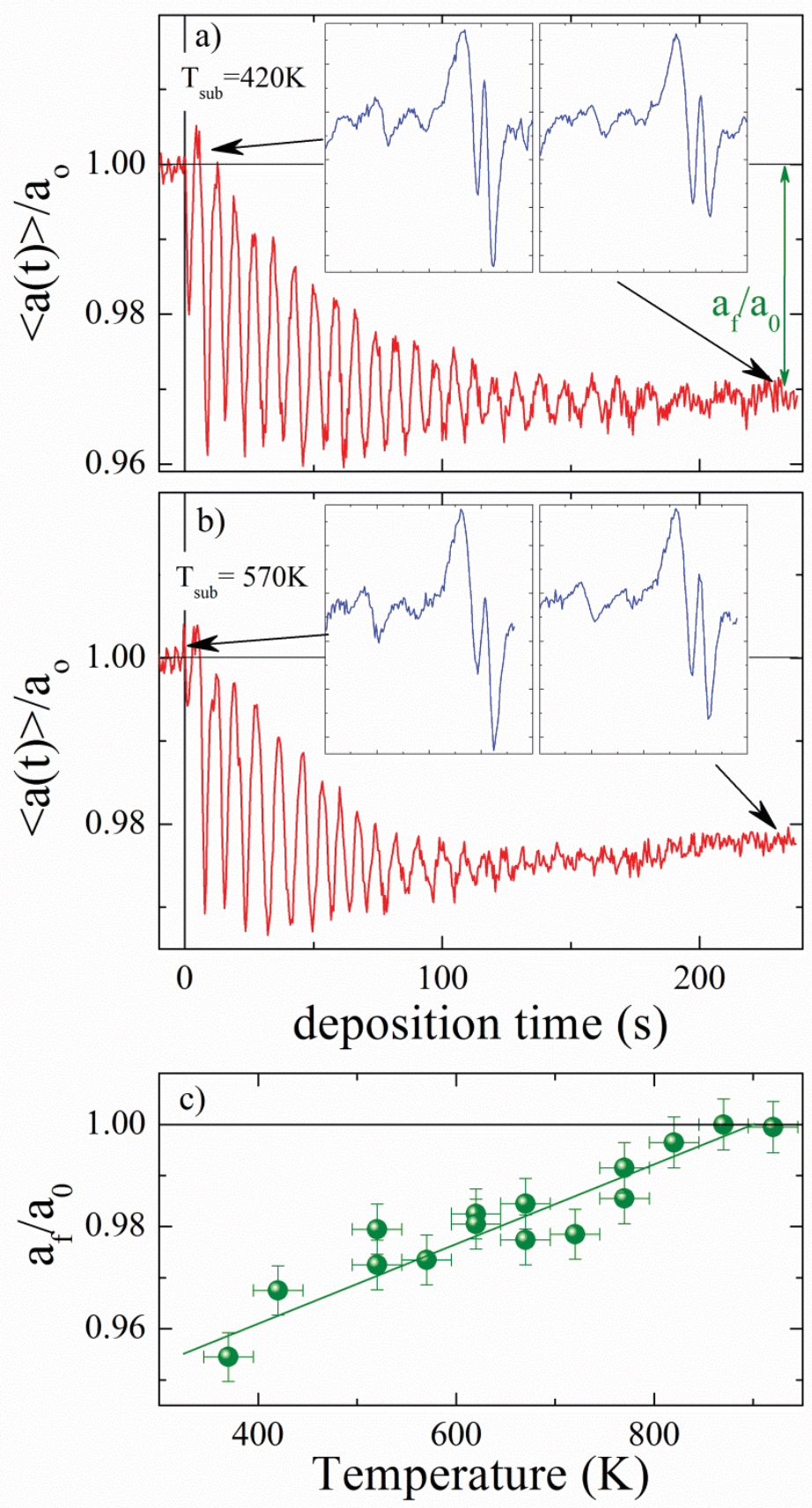

Figure 2 

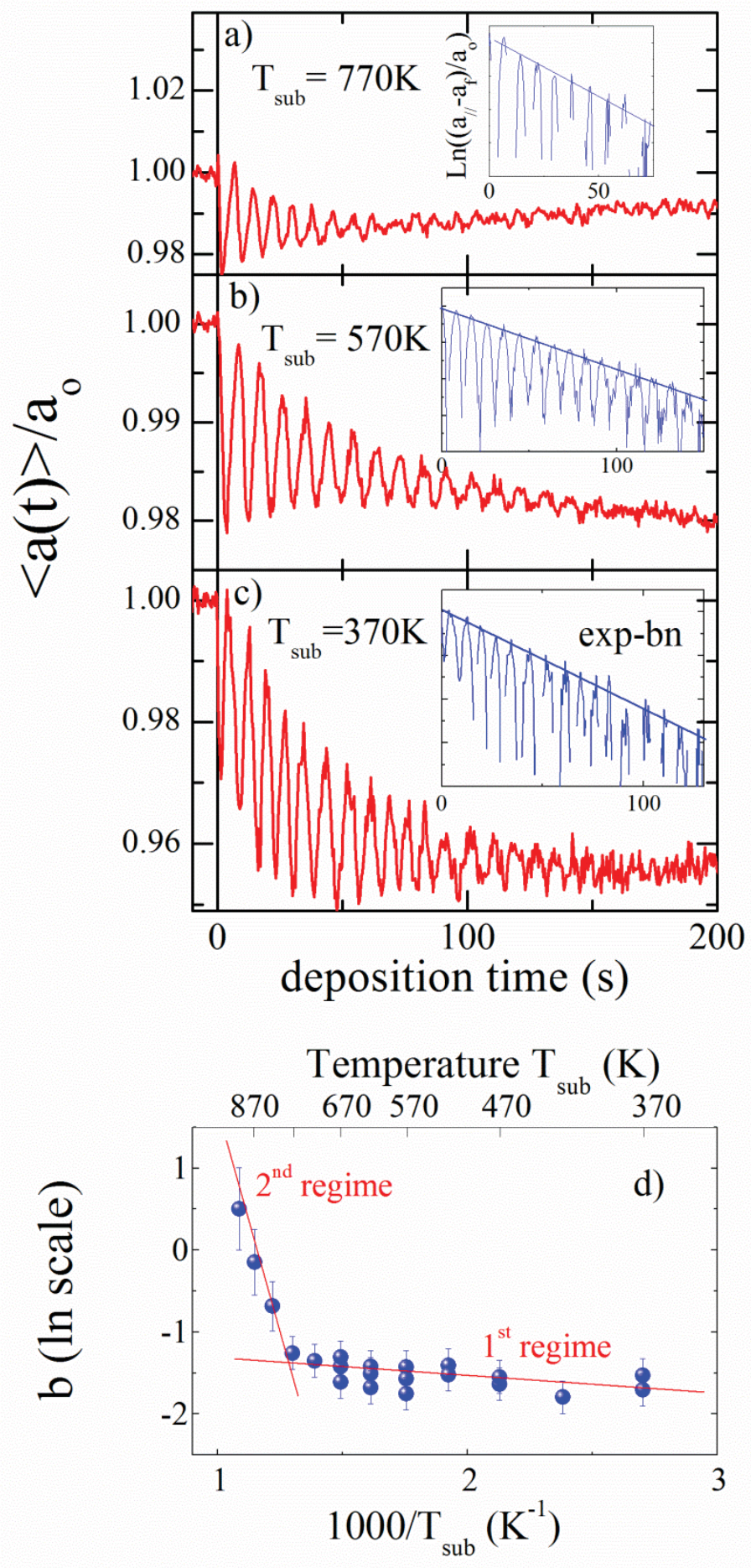

Figure 3 


\section{ACCEPTED MANUSCRIPT}
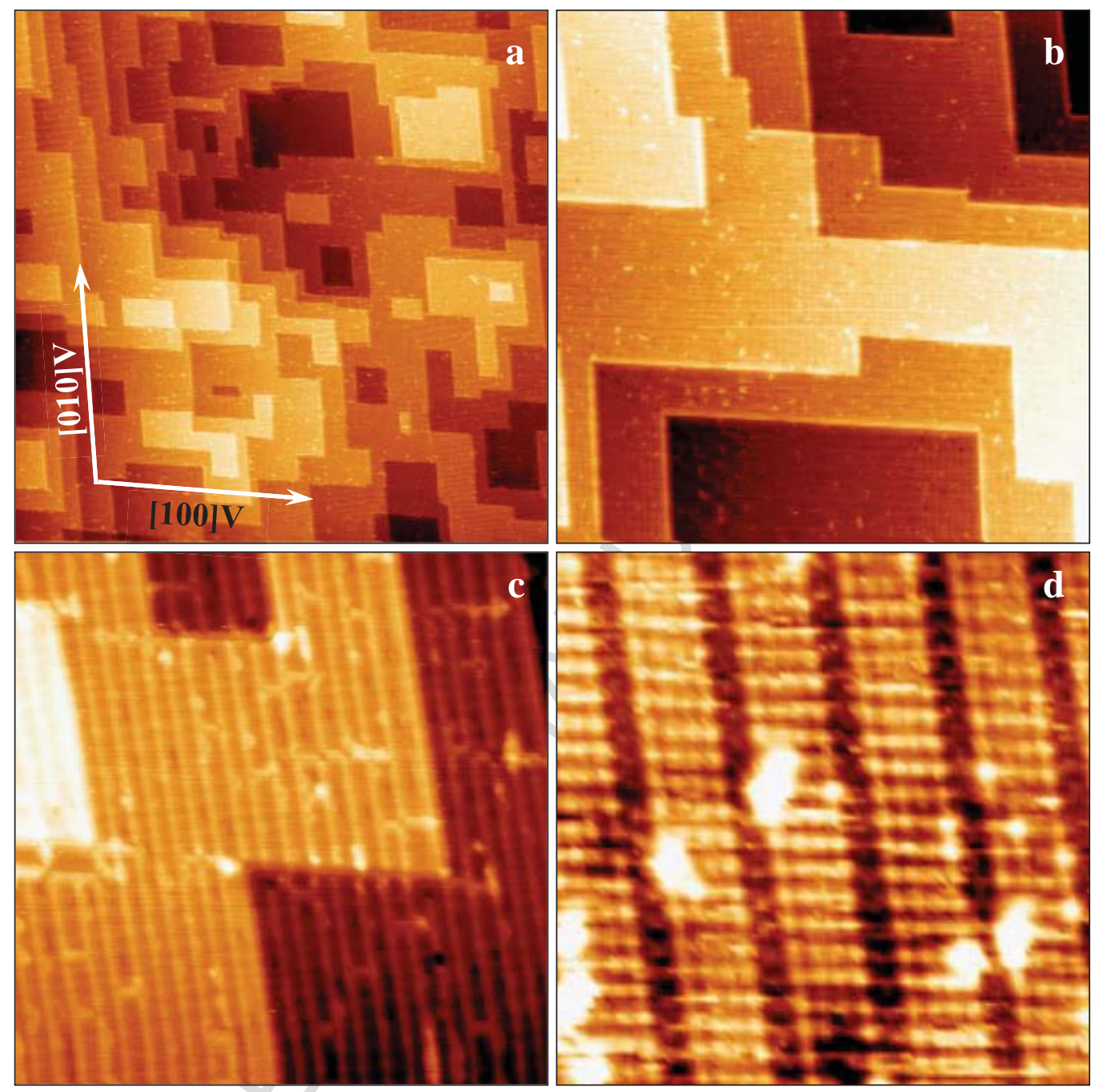

Figure 4 

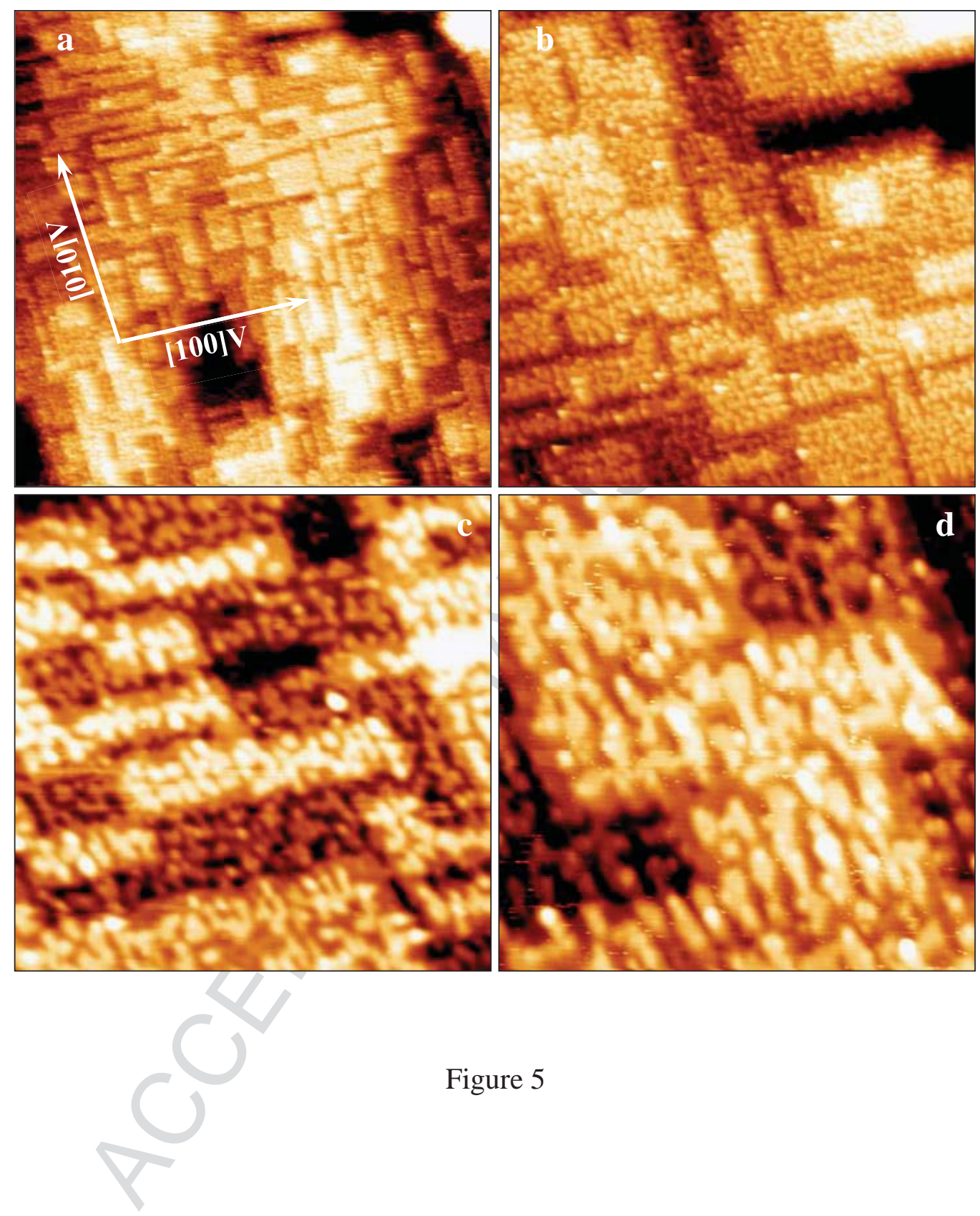

Figure 5 


\section{ACCEPTED MANUSCRIPT}
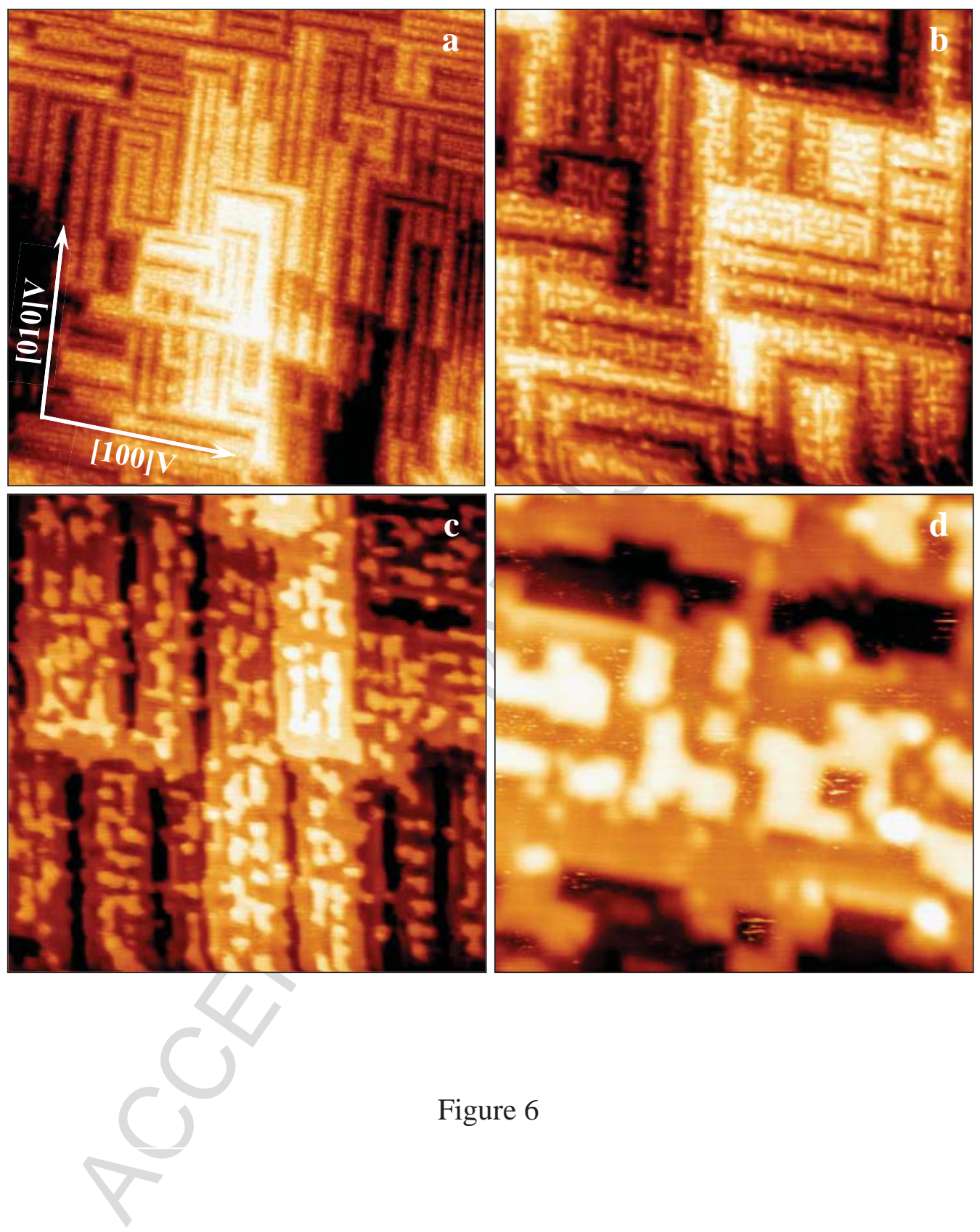

Figure 6 


\section{ACCEPTED MANUSCRIPT}
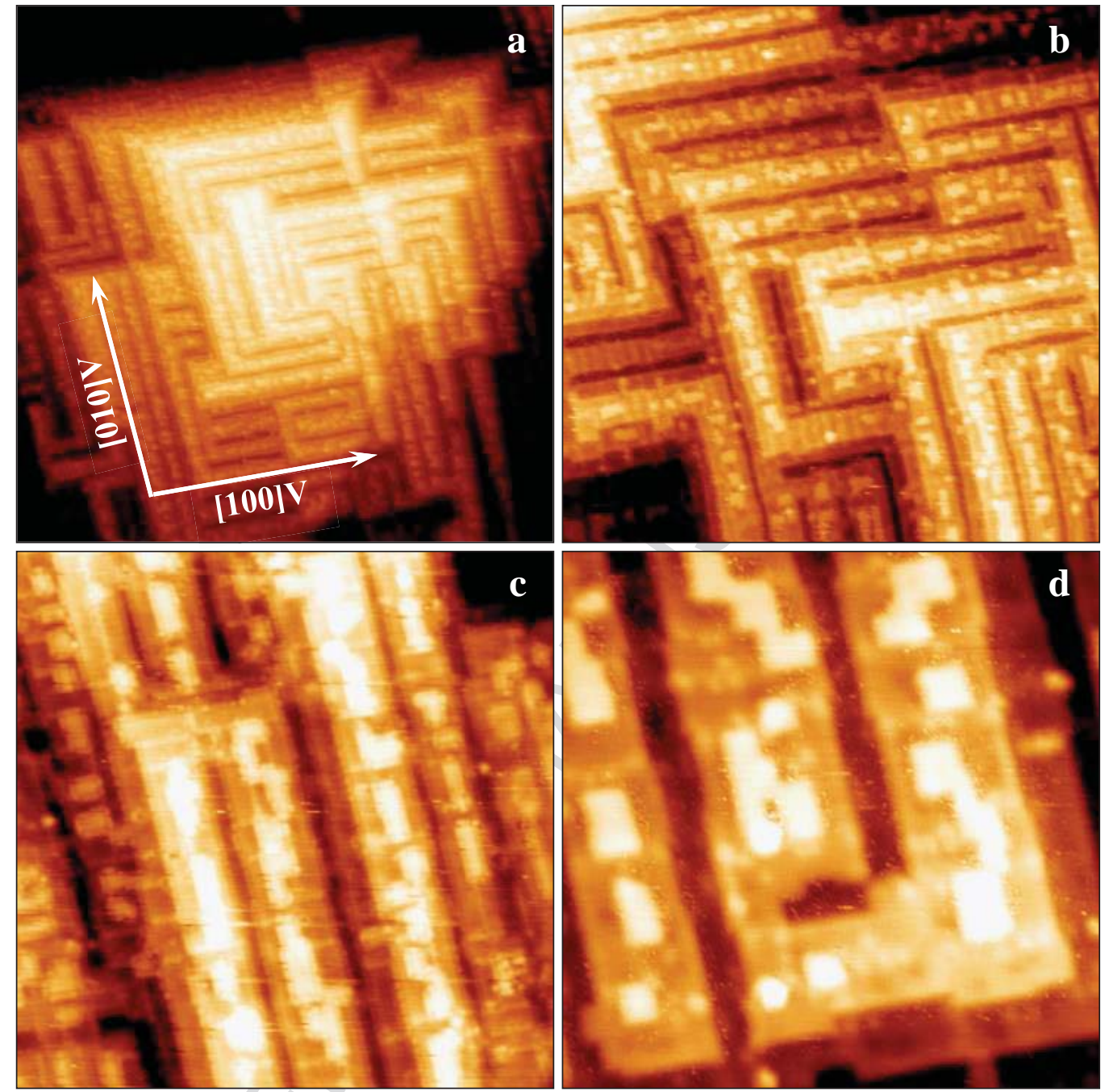

Figure 7 


\section{ACCEPTED MANUSCRIPT}
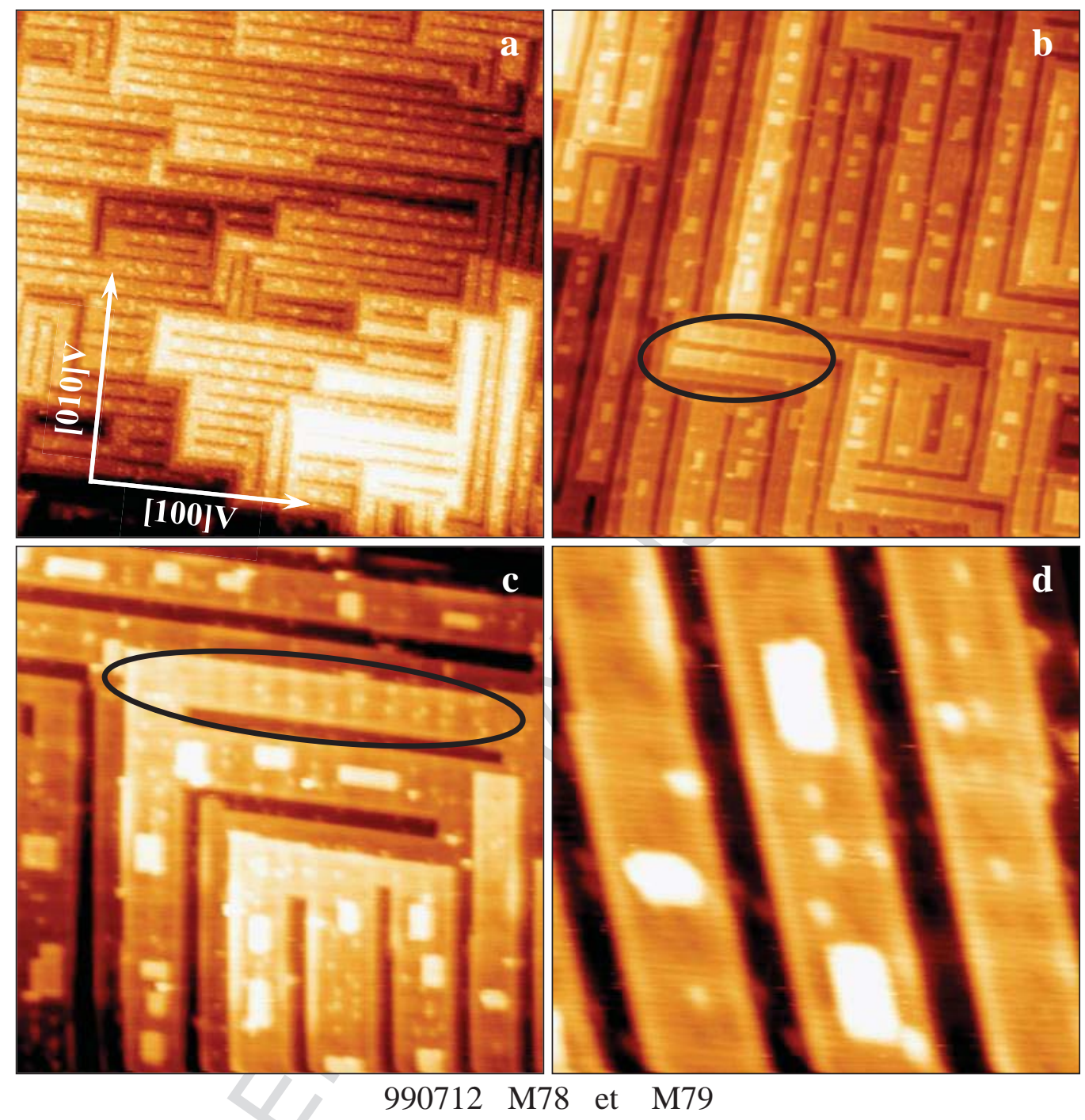

Figure 8 


\section{ACCEPTED MANUSCRIPT}
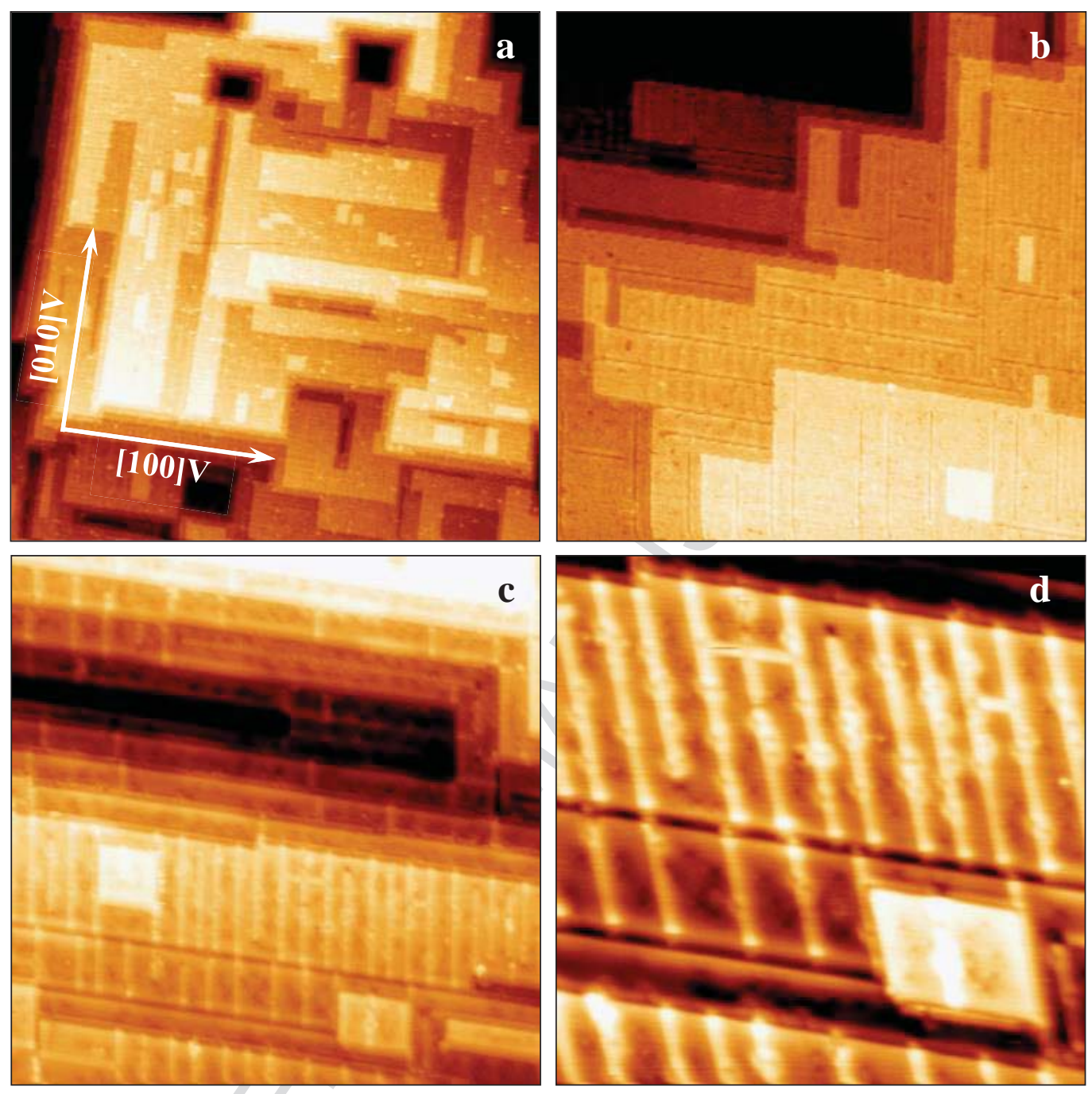

Figure 9 


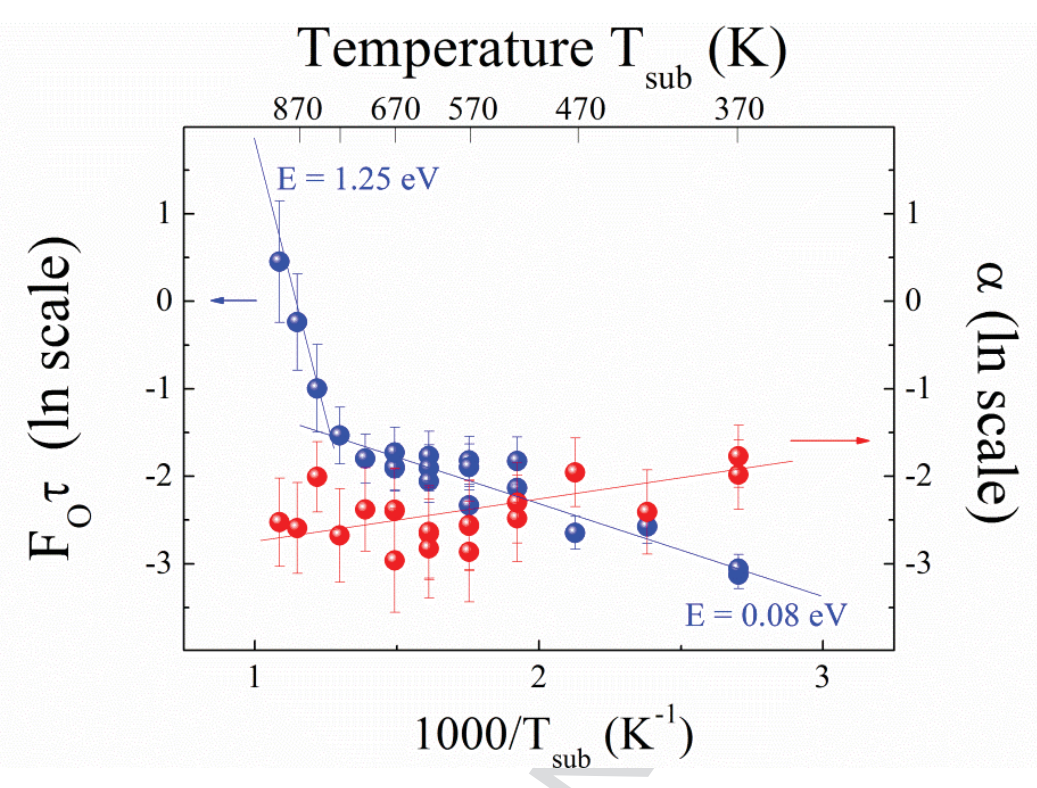

Figure 10: Ln-plot of the oxygen supply $F_{O} \tau$ and incorporation probability $\alpha$ versus the inverse of growth temperature. These data are extracted -see text- from static data $a_{f}$ (fig.2c) and dynamic data $b$ (fig.3d). Two regimes are observed on the oxygen supply given by $F_{O} \tau$ : below $800 \mathrm{~K}$ the oxygen is supplied by incoming flux from the vanadium e-gun, and above $800 \mathrm{~K}$ oxygen upward diffusion from the $\mathrm{V}$ underlayer to the surface becomes efficient. 


\section{ACCEPTED MANUSCRIPT}

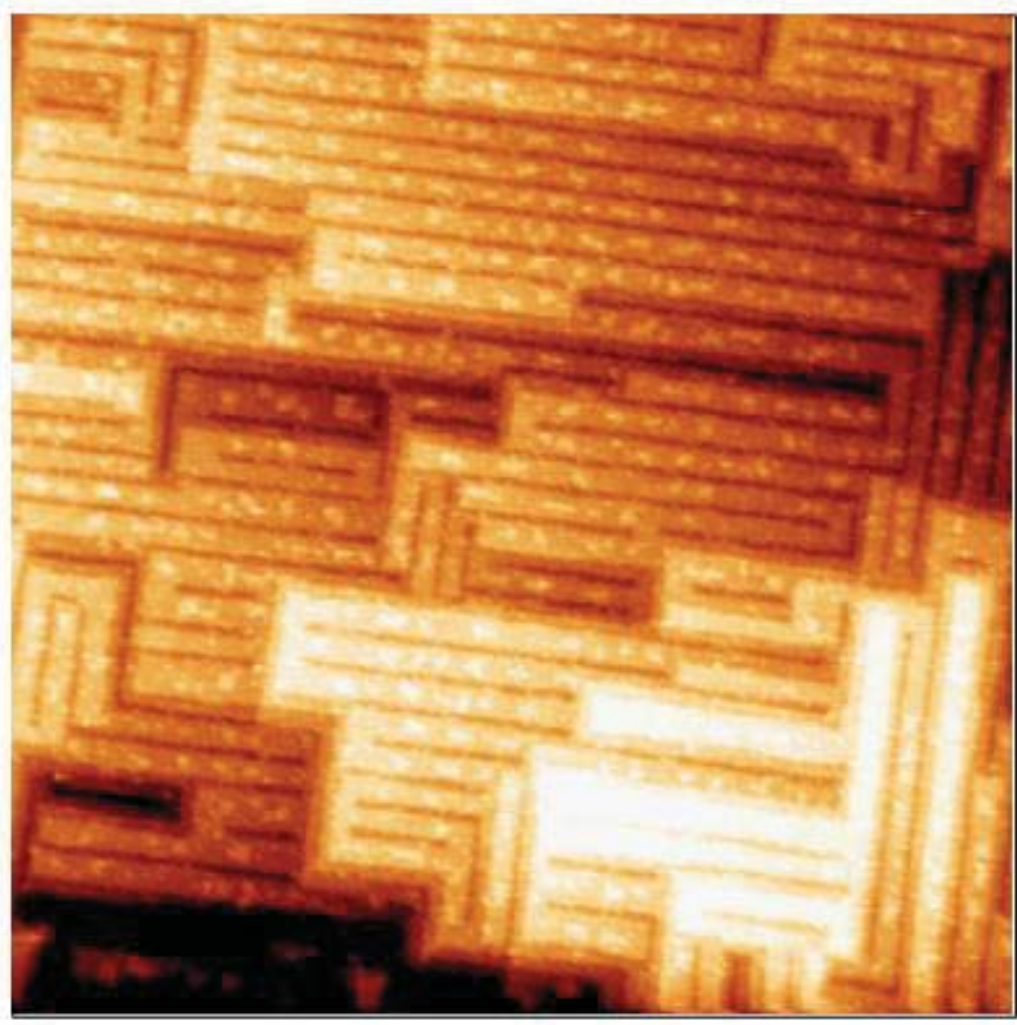

Graphical abstract

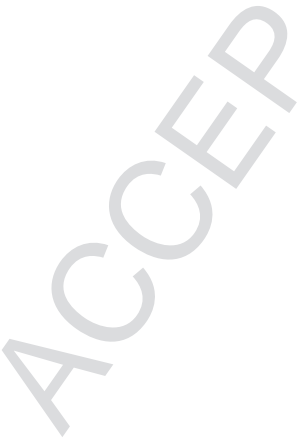




\section{Highlights}

- self-organization during homoepitaxial growth of vanadium in presence of oxygen.

- evidence of oxygen segregation during the growth that play a role in the self-organization

- evidence of strain induced by oxygen that explains the self-organization

- a simple kinetic model accounts for the results 